\title{
Cluster Oriented Optimized Cloud Task Scheduling Strategy using Linear Programming
}

\author{
Mahesh S. Shinde \\ AISSMS COE \\ Pune University, \\ Maharashtra, India
}

\author{
Anilkumar Kadam \\ AISSMS COE \\ Pune University, \\ Maharashtra, India
}

\begin{abstract}
Today, cloud computing has emerged as revolutionary technology in the IT industry. It has provided boost to the area of parallel and distributed computing. Cloud computing deals with on demand allocation of remotely placed computing and storage devices to the cloud users on charge per utilization basis. Cloud service provider (CSP) is responsible for the allocation of cloud resources to consumers of cloud services so that cloud consumers should get satisfied. Thus to gratify cloud users, CSP must has to schedule cloud resources so that it charges minimum amount to users in return for execution of their tasks on cloud resources. In the proposed task scheduling strategy, Fuzzy C-Means (FCM) is used as a clustering technique which is applied complementary to well known optimization technique - Linear Programming. The proposed task scheduling strategy charges minimum amount to user for execution of his tasks within the time specified by him. The results obtained by proposed task scheduling are compared with the results of existing random task scheduling method.
\end{abstract}

\section{Keywords}

Cloud computing; Fuzzy C-Means; Linear programming; task scheduling; cloud resources.

\section{INTRODUCTION}

Cloud computing has brought revolution in today's internet. It has made a huge impact on the internet based services such as e-commerce, banking, social networking etc. Depending of an ownership and accessibility, cloud can be categorized into four types as public, private, community and hybrid cloud. Again cloud services used by cloud users are SaaS (Software as a Service), IaaS (Infrastructure as a service) and $\mathrm{Pa}$ a (Platform as a Service). SaaS provides readymade applications and tools to user, while PaaS provides platforms required for development of applications (or softwares) and IaaS includes hardware components such as processors, storage devices etc [1]. Figure 1 shows an abstract view of cloud computing environment.

Nowadays, smart phones have become inalienable part of people in the modern world. However, processing power of smart phone has limitations since it is insufficient to process large complex applications (tasks) within smart phone. Therefore for processing these complex tasks, there is need to transfer these tasks to cloud which has adequate resources that are having ability to process them [2]. Cloud task scheduler plays crucial role so that cloud resources are allocated efficiently among the cloud users according to their demands. There are common scheduling algorithms such as Random scheduling, First Come First Served scheduling, Priority based scheduling, Shortest Task First scheduling, Round Robin scheduling etc. which can be used by cloud scheduler for scheduling user submitted tasks to cloud resources [3].

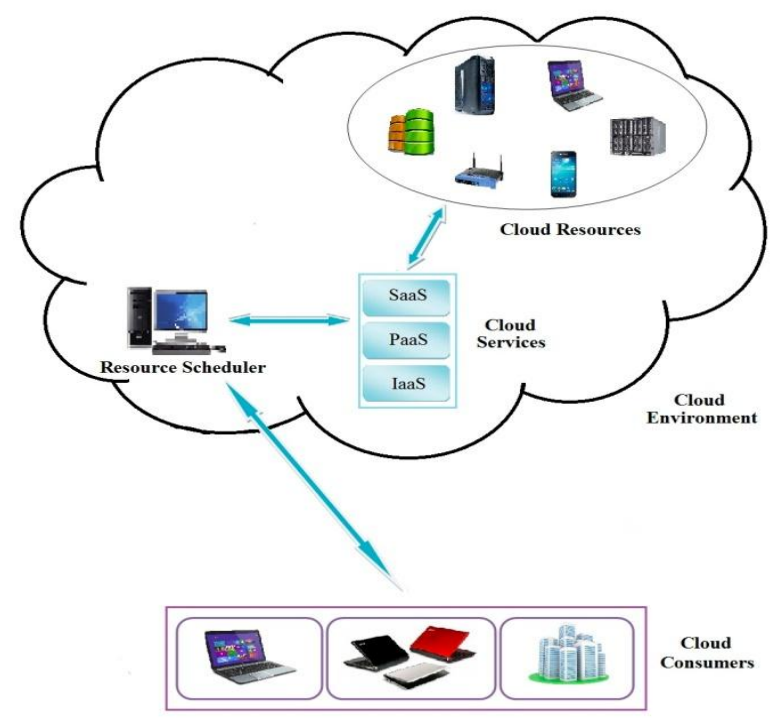

Figure 1: An abstract view of cloud computing

In the proposed system, an approach known as cluster oriented optimized cloud task scheduling strategy using linear programming is used. For this, Fuzzy C-Means (FCM) algorithm has been used as clustering technique complementary to linear programming which is famous optimization technique. In the proposed work, main focus is on the minimization of overall charges (cost) that cloud consumer has to pay to cloud service provider and restricting task executing time within the time limit provided by cloud consumer (task submitter).

\section{RELATED WORK}

Sokol Kosta et al. [2] have proposed a framework which has provision of transferring the smart phone's tasks and applications over the cloud for processing so that the problem of insufficient processing power of smart phones has been resolved. But this framework has not provided any type of task scheduling on cloud.

Zixue Cheng et al. [4] have introduced an architecture having three layers as wearable devices, smart phones and cloud respectively. To mitigate the problem of insufficient processing power of wearable devices, applications or code available at the wearable devices are transferred to smart phone for execution. Again if that smart phone is unable to process, further these tasks are migrated to cloud for 
execution. But this architecture also has not tried any task scheduling at cloud level.Ning Liu et al. [5] have provided framework to accomplish an objective of energy conservation by using proper task scheduling. Tasks are assigned to cloud resources in the decreasing order of their processing power so that resource having high processing power gets highest number of tasks. In this way, user provided bunch of tasks can be executed with minimum number of cloud resources, which in turns minimizes energy consumption.

Sivadon Chaisiri et al. [6] introduced a scheduling strategy with the objective of minimization of overall resource provisioning cost. Resource reservation strategy is cheaper than on-demand resource provision mechanism but requires complex prediction of future resource requirement. This scheduling strategy properly resolves the tradeoffs between reservation and on-demand resource provision strategies

Jignesh Lakhani et al. [7] proposed framework to minimize the communication overhead. This scheduling algorithm sends all the inter-dependent tasks in a batch to the corresponding cloud resource rather than sending each task separately.

R. Vijayalakshmi et al. [8] provided task scheduling framework that deals with minimization of execution time required for user submitted tasks. To achieve a goal of time minimization, task priorities are considered. Task that having highest priority is scheduled to processor with high processing capability. But in this framework, cost minimization problem is not considered.

AV. Karthick et al. [9] have introduced task scheduling based on three queues using Tri Queue Scheduling (TQS). Main objective of this scheduling was to maximize the utilization of available resources thereby improve the performance. Dynamic time slice is used to execute the tasks in small, medium and large sized queues.

Thus nobody from above task scheduling method provided optimum task scheduling in terms of processing time and cost (charges that user has to pay to cloud in returns for execution of his tasks). Therefore the proposed task scheduling strategy is developed to mitigate the problem of cost and time optimization by using Fuzzy C-Means algorithm and linear programming approach. In the proposed system, cost that user has to pay to cloud is minimized under the time limit (constraint) provided by user himself.

\section{PROPOSED FRAMEWORK}

In the proposed system, an approach known as cluster oriented optimized cloud task scheduling strategy using linear programming is introduced. A main objective of proposed system is to schedule the cloud user's task among the available resources in such a way that cloud user requires minimum amount to pay to cloud service provider in returns for execution of tasks submitted by him. The proposed system has another goal of executing these user submitted tasks within time limit provided by him. In this system, batch of images for processing are used as tasks provided by cloud user. This batch of images is given to cloud service provider's task scheduler to schedule them to cloud resources. For this, Fuzzy C-Means (FCM) algorithm has been used as clustering technique complementary to linear programming which is famous optimization technique.

\subsection{Fuzzy C-Means (FCM) Algorithm}

Fuzzy C-Means (FCM) Algorithm is clustering technique in which some data points from the set of data points given to the clustering may belong to two or more clusters simultaneously. In FCM clustering technique, each data point is associated with a set of values called as membership values. Each membership value represents the belongingness of that point with respect to the particular cluster. Membership value of a point with respect to particular cluster is between 0 and 1. Thus the FCM is used to determine these membership values and depending upon these values, each data point is assigned to the particular cluster [10]. This Fuzzy C-Means (FCM) algorithm can be applied using below mentioned steps:

Consider there are $n$ elements, $S=\left\{s_{1}, s_{2}, \ldots . s_{n}\right\}$ to be grouped using FCM in $t$ number of clusters, $C=\left\{C_{1}\right.$, $\left.\mathrm{C}_{2}, \ldots . \mathrm{C}_{\mathrm{t}}\right\}$ then

1. Decide number of final clusters we want from clustering.

2. Initially define random membership value $\mathrm{w}_{\mathrm{ij}}$ (belongingness of point $s_{i}$ to cluster $C_{j}$ )

Where, $i=\{1,2, \ldots \ldots, n\}$ and $j=\{1,2, \ldots ., t\}$

3. Repeat until the difference between membership values in successive iterations is not more than threshold, $\epsilon$.

a) Calculate centroid for each cluster using the formula as follows,

$c_{j}=\frac{\sum_{i=1}^{n} w_{i j}^{p} s_{i}}{\sum_{i=1}^{m} w_{i j}^{p}}$

Where,

$c_{j}=$ centroid of the $j^{\text {th }}$ cluster and

$\mathrm{P}=$ level of fuzziness (which decides the impact of membership value).

b) Calculate membership value of each point with each cluster using following formula,

$w_{i j}=\frac{\left(1 / \operatorname{dist}\left(s_{i}, c_{j}\right)^{2}\right)^{\frac{1}{p-1}}}{\sum_{q=1}^{t}\left(1 / \operatorname{dist}\left(s_{i}, c_{q}\right)^{2}\right)^{\frac{1}{p-1}}}$

4. Thus finally we will get final centroids and corresponding clusters. [10][11]

\subsection{Linear Programming}

Linear programming is famous optimization approach used in business. Linear programming deals with the linear equations that represent the system under consideration. For applying linear programming, the system is represented in three parts, namely, objective function (equation of particular parameter which is to be optimized), constraints (equations that represent some limits while optimizing the objective function) and non-negativity constraints (list of parameters which can't be negative). A typical representation of linear programming equations is as follows [12]: 
Maximize (or Minimize),

$$
z=\sum_{j=1}^{n} a_{j} x_{j}
$$

Subject to:

$$
\sum_{j=1}^{n} b_{i j} x_{j}
$$

$$
\text { for } \mathrm{i}=1,2,3, \ldots \ldots, \mathrm{m}
$$

Non-negativity constraints:

$$
x_{j} \geq 0
$$

$$
\text { for } \mathrm{j}=1,2,3, \ldots \ldots, \mathrm{n}
$$

where,

$a_{j}, b_{i j}$ and $c_{i}$ are set of real numbers and $x_{j}$ are set of variables.In this way, the objective of linear programming is to find out the value of $x_{i}$ in such a way that objective function will get optimized (minimized or maximized) without violating any constraint. For solving the linear equations, generally Simplex algorithm is used. A Simplex algorithm is as follows [13]:

Step 1: Construct linear equations in standard form for given problem.

Step 2: Transform linear equations into slack form in order to convert inequality constraints into equality constraints by adding slack variables

Step 3: Calculate a basic solution by making all the nonbasic variables (variables present on right hand side) to 0 and then finding the values of all basic variables (variables present on the left hand side)

Step 4: Rewrite the set of equations and reform linear equations until basic solution changes in each iteration and gives greater objective value as compared to previous iteration. Following steps are carried out for this goal:

1) Select a non-basic variable having positive coefficient in objective function.

2) Until no constraint is violated, expand the value of this chosen non-basic variable.

3) Then change this non-basic variable to basic variable and thereby existing basic variable to non-basic variable.

Step 5: Until all coefficients in the objective function become negative, step 3 and 4 are repeated.

Step 6: Now required objective value is obtained just by putting final basic solution in original linear equations.

\subsection{System Architecture}

Proposed system presents cluster oriented optimized cloud task scheduling strategy using linear programming algorithm. The proposed system has used computers and smart phones as cloud resources which are allocated to process submitter's tasks by charging different costs to user according to their processing power. Generally, resources having high processing power (computers) charges more cost to user as compared to resources that having low processing power (smart phones). But as computers having high processing power, they execute tasks or applications in less time than smart phones. Therefore it is important to schedule submitter's tasks in such a way that it optimizes cost charged to submitter (cloud user) and time required to execute these tasks. Architecture of this proposed task scheduling strategy is depicted in figure 2. As shown in architecture, cloud consumer (submitter) submits group of images to cloud for processing (grey scaling). Cloud scheduler receives this batch of images from submitter and applies Fuzzy C-Means (FCM) in order to bundle them together according to their sizes into three groups, namely, small, medium and large. Out of these, small and large sized images are as it is given to smart phone (having low processing power) and computer (having high processing power) respectively for processing. And on remaining medium images, linear programming is applied to determine whether a particular image should be given to smart phone or computer for processing as shown in figure 2.

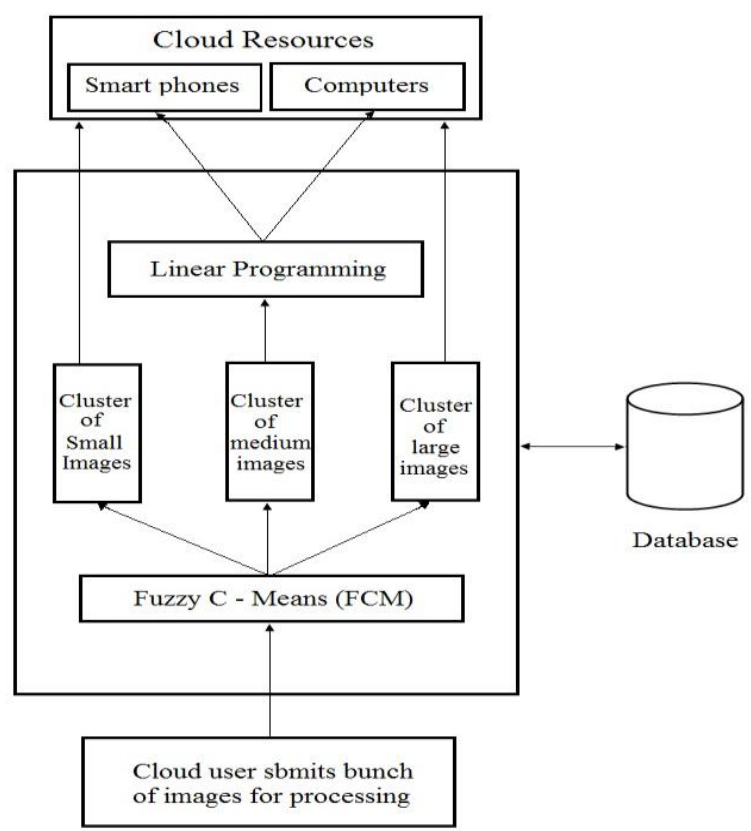

Figure 2: Proposed cluster oriented task scheduling using Linear Programming

As linear programming is to be applied on group of medium sized images, firstly there is need to express this task scheduling system in the form of linear equations required for linear programming. Proposed system is represented in the form of linear equations as follows:

\section{Objective function:}

Minimize,

$$
z=\sum_{i=1}^{n} c_{1 i} x_{1 i}+\sum_{j=1}^{m} c_{2 j} x_{2 j}
$$

Subject to:

$$
\sum_{i=1}^{n} t_{1 i} x_{1 i}+\sum_{j=1}^{m} t_{2 j} x_{2 j} \leq t_{\max }
$$




$$
\sum_{i=1}^{n} x_{1 i}+\sum_{j=1}^{m} x_{2 j}=x_{\text {total }}
$$

Non-negativity constraints:

$$
x_{1 i}, x_{2 j} \geq 0
$$

Where,

$\mathrm{c}_{1 \mathrm{i}}=$ cost per pixel for $\mathrm{i}^{\text {th }}$ smart phone

$c_{2 j}=$ cost per pixel for $j^{\text {th }}$ computer

$\mathrm{x}_{1 \mathrm{i}}=$ total number of pixels processed by $\mathrm{i}^{\text {th }}$ smart phone

$\mathrm{x}_{2 \mathrm{j}}=$ total number of pixels processed by $\mathrm{j}^{\text {th }}$ computer

$\mathrm{t}_{1 \mathrm{i}}=$ processing time per pixel at $\mathrm{i}^{\text {th }}$ smart phone

$t_{2 j}=$ processing time per pixel at $j^{\text {th }}$ computer

$\mathrm{t}_{\max }=$ maximum time allowed to process the entire batch of images

$\mathrm{x}_{\text {total }}=$ total number of pixels in the medium sized images

Thus goal is to minimize the total cost as represented in objective function within the minimum or specified time. Now, we use Simplex algorithm to solve this linear problem to find values of $x_{1 i}$ and $x_{2 j}$. Putting these values in the objective function gives the total minimum cost required to process the batch images within user specified time $t_{\max }$.

\section{EXPERIMENTAL SETUP}

Private cloud is used for a demonstration of the proposed system. One smart phone is used as a cloud user (task submitter) which submits the bunch of images for processing to the task scheduler of this cloud. As shown in figure 3, number of smart phones and computers are used as cloud resources for executing the user submitted tasks (batch of images).Smart phone with Android 4.3 operating system and $1 \mathrm{GHz}$ dual core processor has been used as task submitter. Task submitter module in smart phone of cloud user is developed using eclipse (ADT bundle). The computer with $4 \mathrm{~GB}$ RAM, 1 TB hard disk and $2.6 \mathrm{GHz}$ processor is used as a task scheduler which use GlassFish server. Scheduler module has executed on this computer so that it acts as scheduler (CSP) of system. Image processing (gray scaling of images) logic is implemented on number of computers and smart phones which were used as cloud resources. For the sake of evaluation of the proposed task scheduling, the charge of processing for smart phone and computer resources are fixed to five and ten units per thousand pixels respectively.

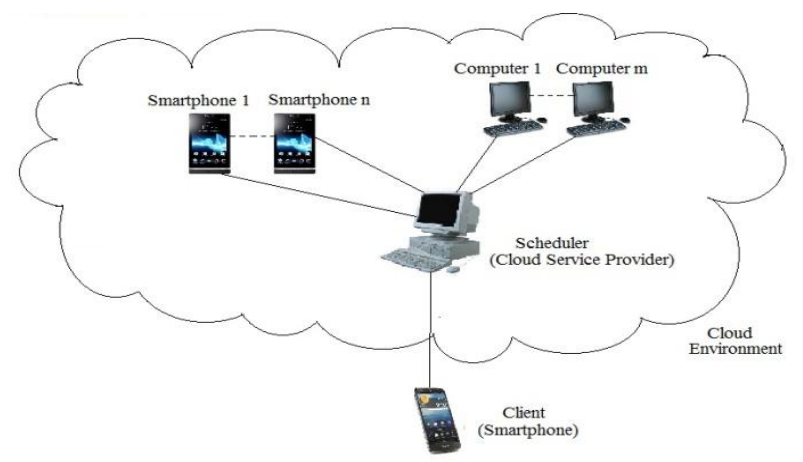

\section{EXPERIMENTAL RESULTS}

The evaluation of proposed task scheduling has been done by comparing the results (in terms of charges user required to pay to CSP) obtained by submitting same set of images to random task scheduling and proposed task scheduling. As linear programming has been used in the proposed system for task scheduling, cost required for the processing of input group of images depends on the user submitted time (time constraint). As computer resource has high processing capability than that of smart phone resource, time required for computer to process submitted tasks is shorter than smart phone while cost required is high as compared to smart phone. Therefore the proposed system tries to optimize time and cost depending on the user permitted time bound. Results of proposed scheduling strategy have been obtained under tight time bound (giving less time for execution) and relaxed time bound (allowing more time for execution) provided by user. Following table 1 shows the comparison of results obtained by random task scheduling and proposed task scheduling strategy (in tight and relaxed time bound).

Table 1: Charge (cost) comparison of random and proposed task scheduling system

\begin{tabular}{|c|c|c|c|c|}
\hline $\begin{array}{c}\text { Sr. } \\
\text { No. }\end{array}$ & $\begin{array}{c}\text { Number } \\
\text { of } \\
\text { images }\end{array}$ & $\begin{array}{c}\text { Charges in } \\
\text { random } \\
\text { task } \\
\text { scheduling }\end{array}$ & \multicolumn{2}{|c|}{$\begin{array}{c}\text { Charge in proposed task } \\
\text { scheduling strategy }\end{array}$} \\
\cline { 3 - 5 } & & $\begin{array}{c}\text { Tight } \\
\text { time } \\
\text { bound }\end{array}$ & $\begin{array}{c}\text { Relaxed } \\
\text { time bound }\end{array}$ \\
\hline 1 & 3 & 3290 & 3150 & 2910 \\
\hline 2 & 6 & 6720 & 5810 & 5135 \\
\hline 3 & 9 & 9130 & 9060 & 7960 \\
\hline 4 & 12 & 10940 & 10780 & 8820 \\
\hline 5 & 15 & 15280 & 13560 & 12835 \\
\hline
\end{tabular}

Charge (cost) graph of random task scheduling and proposed task scheduling system under tight and relaxed time bound is shown in Figure 4.

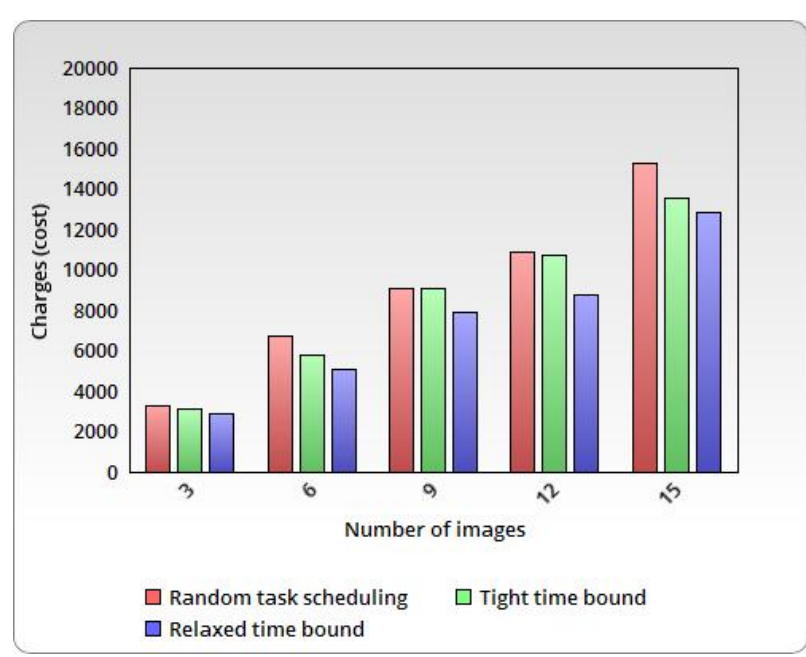

Figure 4: Cost graph of Random and proposed task scheduling (under tight and relaxed time bound)

Figure 3: Experimental setup for proposed system 
Graph in the Figure 4 shows that the cost required to execute the user submitted tasks is optimized on the basis of user specified time constraint for task execution.Execution time comparison in case of tight and relaxed time bound of the proposed system is given in table 2 . These values of processing time (in seconds) of number of images are obtained when user specifies tight and relaxed time bound for processing of these images.

Table 2: Execution time comparison proposed system under tight and relaxed time bound

\begin{tabular}{|c|c|c|c|}
\hline \multirow{2}{*}{$\begin{array}{c}\text { Sr. } \\
\text { No. }\end{array}$} & $\begin{array}{c}\text { Number of } \\
\text { images }\end{array}$ & \multicolumn{2}{|c|}{$\begin{array}{c}\text { Time required to process } \\
\text { images in the proposed task } \\
\text { scheduling strategy (in seconds) }\end{array}$} \\
\cline { 3 - 4 } & & $\begin{array}{c}\text { Tight time } \\
\text { bound }\end{array}$ & $\begin{array}{c}\text { Relaxed time } \\
\text { bound }\end{array}$ \\
\hline 1 & 3 & 24 & 55 \\
\hline 2 & 6 & 45 & 73 \\
\hline 3 & 9 & 67 & 96 \\
\hline 4 & 12 & 85 & 117 \\
\hline 5 & 15 & 110 & 146 \\
\hline
\end{tabular}

Comparison of execution (processing) times of number of images under the user submitted tight and relaxed time bounds is shown by graph as shown in Figure 5. Now by comparing Figure 4 and Figure 5, it is observed that if user gives tight time bound for execution of images (when execution time decreases) results in increase in cost (charge) of processing. And if user allowed time bound is larger (under relaxed time bound) then charges required to pay to the cloud service provider are low. Therefore proposed task scheduling strategy allows user to control the execution of his tasks on cloud resources by giving time bounds based on his requirement that whether he want to process his tasks in minimum time or minimum cost.

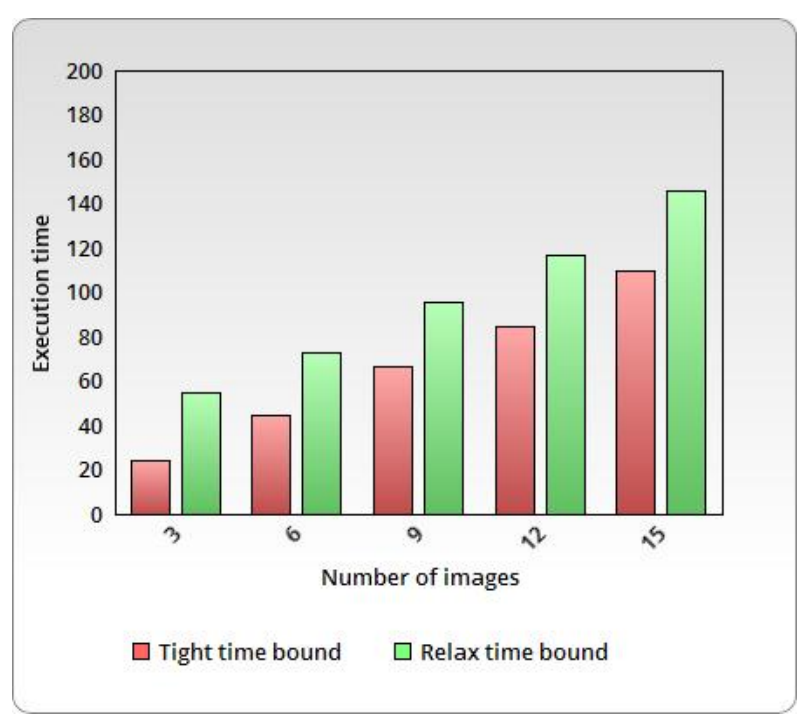

Figure 5: Execution time graph under tight and relaxed time bound of proposed system

\section{CONCLUSION}

Today, cloud computing is becoming more popular which has resulted in increasing number of cloud users day by day. Therefore the role of task scheduler is very important to manage shared resources among cloud consumers in order to attain proper resource utilization, load balancing, user satisfaction by cost and time optimization, energy conservation etc. This paper has introduced cluster oriented optimized cloud task scheduling strategy using linear programming approach with an objective of cost and time optimization. As shown in results, using the proposed task scheduling strategy, one can minimize charges that user required to pay in return for processing his task on cloud resources within the time limit supplied by him.

\section{FUTURE SCOPE}

The proposed system has used the linear programming approach for the task scheduling to achieve the objective of cost (charges) minimization under time user specified time bound. Depending upon user's requirement, it is also possible to design linear programming by reversing the parameters for objective function and constraints as time and cost respectively. Also some more number of parameters like resource utilization, communication cost, energy consumption etc. can be used while constructing linear programming equations to make scheduling strategy more extensive.

\section{REFERENCES}

[1] Borko Furht and Armando J. Escalante, Handbook of Cloud Computing, ISBN 978-1-4419-6523-3, Springer, 2010.

[2] Sokol Kosta, Andrius Aucinas, Pan Hui, Richard Mortier and Xinwen Zhang, "ThinkAir: Dynamic resource allocation and parallel execution in the cloud for mobile code offloading", Proceedings IEEE INFOCOM, IEEE 978-1-4673-0775-8/12, PP: 945 $953,2012$.

[3] Charles Crowley, "Operating Systems: A DesignOriented Approach”, McGraw-Hill, November 1996.

[4] Zixue Cheng, Peng Li, Junbo Wang and Song Guo, "Just-in-Time Code Offloading for Wearable Computing", IEEE Transactions on Emerging Topics in Computing, 2015.

[5] Ning Liu, Ziqian Dong and Roberto Rojas-Cessa, "Task Scheduling and Server Provisioning for EnergyEffcient Cloud-Computing Data Centers", IEEE 33rd International Conference on Distributed Computing Systems Workshops, IEEE 978-0-7695-5023-7/13, PP: 226-231, 2013.

[6] Sivadon Chaisiri, Bu-Sung Lee and Dusit Niyato, "Optimization of Resource Provisioning Cost in Cloud Computing", IEEE TRANSACTIONS ON SERVICES COMPUTING, IEEE 1939-1374/12, PP: 164-177, 2012.

[7] Jignesh Lakhani and Hitesh A. Bheda, "An Approach to Optimized Resource Scheduling using Task Grouping in Cloud", International Journal of Advanced Research in Computer Science and Software Engineering, ISSN:2277 128X, Volume 3, Issue 9, September 2013, PP: 594-599.

[8] R. Vijayalakshmi and Mrs. Soma Prathibha, "A novel approach for task scheduling in cloud", 4th ICCCNT, IEEE-31661, July 4-6, 2013.

[9] AV. Karthick, Dr. E. Ramaraj and R. Kannan, “An Efficient Tri Queue Job Scheduling using Dynamic 
Quantum Time for Cloud Environment”, International Conference on Green Computing, Communication and Conservation of Energy (ICGCE), IEEE 978-1-46736126-2/13, 2013.

[10] Paan-Ning Tan, Michael Steinbach and Vipin Kumar, Introduction to Data Mining, Pearson Education, ISBN 978-81-317-1472-0, 2013.

[11] YIN Zhonghang, TANG Yuangang, SUN Fuchun and SUN Zengqi, "Fuzzy Clustering with Novel Separable
Criterion", TSINGHUA SCIENCE AND TECHNOLOGY, ISSN 1007-0214, Volume 11, Number 1, PP: 5053, February 2006.

[12] John W. Chinneck, Practical Optimization: A Gentle Introduction, 2001, [Online] Available at: http://www.sce.carleton.ca/faculty/chinneck/po.html

[13] Thomas H. Cormen, Charles E. Leiserson, Ron Rivest, Clifford Stein, "Introduction To Algorithms", MIT Press, 2001 